\title{
Signal Processing of Internet of Vehicles Based on Intelligent Interference
}

\section{Xiangyu Wu ( $\nabla$ 593200637@qq.com )}

Harbin Engineering University https://orcid.org/0000-0001-7959-3002

\section{Changbo Hou}

Harbin Engineering University

\section{Zhian Deng}

Harbin Engineering University https://orcid.org/0000-0002-1200-8697

\section{Chenyu Fang}

Harbin Engineering University

Guowei Liu

Harbin Engineering University

\section{Research Article}

Keywords: oV signal processing, Intelligent interference, Convolutional autoencoder, Interference waveform generation

Posted Date: January 19th, 2022

DOI: https://doi.org/10.21203/rs.3.rs-1263552/v1

License: (c) (i) This work is licensed under a Creative Commons Attribution 4.0 International License.

Read Full License 


\title{
Signal Processing of Internet of Vehicles Based on Intelligent Interference
}

\author{
Xiangyu Wu? ${ }^{\text {? }}$, Changbo Hou??,??, Zhian Deng??,????, Chenyu Fang??, Guowei Liu??
}

\author{
?? Correspondence: ?? \\ ?? College of Information and \\ Communication Engineering, \\ Harbin Engineering University, \\ Harbin, China \\ Full list of author information is \\ available at the end of the article
}

\begin{abstract}
With the rapid development of in-vehicle electronic technology and artificial intelligence, Internet of Vehicles (loV) technology, as an effective integration of the two, greatly reduces the probability of road traffic accidents. However, the current loV system is not perfect for the control process of abnormal vehicles. Therefore, in order to strengthen the management and control of abnormal vehicles in the loV, it is extremely necessary to propose a method for interfering with loV signals. Among the current popular intelligent interference methods, most of them rely on the prior knowledge of the signal to deduce the best interference waveform. However, These methods rely too much on prior knowledge to be practically applicable. Therefore, in view of the shortcomings of the current communication interference technology, this study proposes an interference waveform generation technology based on convolutional autoencoders. The convolutional autoencoder was used to change the features on the fully connected layer to generate an interference waveform that is very similar to the received signal waveform, and the interference waveform is sent to the receiver to realize the control of the loV signal. The simulation results show that the interference waveform generation technology proposed in this study can make the bit error rate (BER) reach $38.4 \%$ within the signal-to-interference ratio (SIR) from $-10 \mathrm{~dB}$ to $-15 \mathrm{~dB}$.
\end{abstract}

Keywords: loV signal processing; Intelligent interference; Convolutional autoencoder; Interference waveform generation

\section{Introduction}

With the development of artificial intelligence technology, in the future 6G era, various network models of deep learning will be ubiquitous. One of the most critical scenarios is that service providers need to interact and make decisions on vehicle information in real time [?, ?].

$\mathrm{IoV}$ is based on the Internet of Things [?, ?] technology and is more oriented to transportation applications. At present, the IoV can obtain environmental information around the vehicle through the image sensor on the vehicle [?, ?]. This information processing technology has greatly improved traffic safety. However, the current signal processing technology of the IoV is not perfect. When the owner deliberately violates the rules or the vehicle sensing system fails, it is difficult to avoid traffic accidents only by relying on the subjective initiative of the owner. Therefore, safe, effective and intelligent interference to illegal driving behavior is an important field of IoV signal control. IoV signal control is based on IoV signal processing. Therefore, to improve the abnormal IoV signal control technology, 
this study introduces intelligent interference technology into the field of IoV signal processing.

At present, interference methods for digital communication are still dominated by traditional interference methods, which hinder the target receiver from receiving information through high-power suppression. However, the density of traffic flow on urban roads is relatively large, and the number of vehicles with abnormal conditions is often a small number. High-power barrage jamming cannot distinguish specific interference targets, which means that traditional interference methods are not suitable for IoV signal processing. Therefore, the introduction of intelligent communication interference into IoV signal processing has become a major trend in the development of the current IoV field.

Research on intelligent interference has been carried out for several years at home and abroad. Traditional methods are generally based on optimization theory, information theory, game theory [?] and other technologies. They rely on prior knowledge and combine signal analysis optimization and game theory to obtain optimal interference strategies and optimal interference waveforms under specific constraints. References [?, ?, ?] analyze the capacity of wireless channels in the presence of correlated interference, and prove that Gaussian signaling and Gaussian interference form a saddle-point solution. In 2016, Scholars such as Amuru deduced the theoretical optimal interference waveforms for several classical digital modulations, and proved that it is not optimal to match the jammer's signal with the disturbed signal to obtain the maximum BER probability. These interference methods based on optimization theory need to have certain prior knowledge of the modulation method of the disturbed signal and the communication network environment, so that the corresponding optimal interference waveform can be accurately analyzed. When the environment changes, the algorithm often cannot adapt quickly according to the parameter changes of the target.

Thanks to the rapid development of computer equipment, scholars have begun to study the interference algorithm of artificial intelligence. The work done at home and abroad mainly focuses on artificial intelligence algorithms, makes autonomous decisions in terms of interference strategies, and finds the best interference pattern under the existing interference waveform pattern library. Amuru et al. [?] proposed to use a multi-armed bandit machine to model the interference waveform decision problem, and studied the interference strategies for one-to-one and many-to-many scenarios in the absence of prior information. At the same time, continuous and discrete action state spaces are established, which provides a reference for subsequent modeling and exploration based on reinforcement learning methods. Yang H.J. et al. applied the reinforcement learning algorithm to the selection of the interference channel, and explored the action space based on the power and signal modulation method through the reinforcement learning network, so as to achieve the purpose of tracking the dynamic interference of the enemy target. Zhang J.Y. searched the entire strategy combination space by means of Gaussian perturbation exploration, generates the optimal interference strategy, and improved the selection speed of the optimal interference strategy. Wu Z.G. et al. proposed reinforcement learning methods based on state-action-reward-state-action (SARSA) and Q-learning, optimized the design of interference waveforms, and verified the feasibility of the fill 
collaborative forecast and replenishment (Fill-CFAR) method of interference design. Zhang X.M. et al. established a Markov chain dynamic interference model and used reinforcement learning to design interference waveforms with various exploration strategies.

However, these methods above rely too much on prior information, and in practical application scenarios, it is very difficult to obtain prior information of the disturbed signal.

Therefore, for the case of unknown prior information, this study proposes a method based on convolutional autoencoder to parse the received signal and generate an interference waveform with similar characteristics to the original signal. In this study, we choose to interfere with the communication signals of three modulation methods: binary phase shift keying (BPSK), quaternary phase shift keying (QPSK) and octal phase shift keying (8PSK) . After denoising, the signal sent by the transmitter is input into the convolutional auto-encoder to extract signal features, and the interference waveform is generated by changing the parameters of the fully connected layer, and the interference effect is evaluated at the receiver. This study makes the following three contributions to the problem of signal management and control of the IoV:

1) The method used in this study does not rely on prior knowledge.

2) The shape of the interference waveform generated by the method used in this study is very similar to the shape of the disturbed signal waveform, so that the receiver cannot judge whether the interference is applied or not.

3) The interference effect of the method adopted in this study is superior to Gaussian noise interference and amplitude modulation (AM) noise interference under any SIR. When the signal-to-noise ratio(SNR) is $15 \mathrm{~dB}$, and the SIR is within $-10 \mathrm{~dB}$ to $-15 \mathrm{~dB}$, the interference waveform generation technology proposed in this study can make the bit error rate reach $38.4 \%$.

This study is organized as follows: Section 2 presents the method used in this study, and describes the model and algorithm network structure of the optimal interference waveform generation based on convolutional autoencoders proposed in this study. Section 3 presents the simulation results of the experimental simulation. Section 4 draws conclusions and summarizes future research directions.

\section{Methods}

\subsection{System model}

Traditional interference generally selects the interference waveform that best matches the characteristics of the received signal from the strategy library to impose interference. However, for the signal of the unknown system, it is impossible to find a suitable interference method, and generally barrage interference is used to interfere with the other party. However, the barrage interference has the problem that the required power is too large and cannot be accurately interfered. Therefore, this study proposes a structure based on the convolutional autoencoder to extract features from the signal, and adds perturbations to the features of the fully connected layer to generate interference signals with similar high-level abstract features to the target signal. 


\subsubsection{Model of Interference Waveform Generation Based on Autoencoder}

When the modulation mode adopted by the communication signal is known, the probability density function of its efficient interference can be derived by mathematical formula, and the interference signal can be generated according to the distribution characteristics. However, in the absence of prior knowledge of communication signals, signals with similar characteristics in time domain, frequency domain, information domain, energy domain and feature domain can still be considered as efficient interference. Therefore, this proposes a feature extraction method based on convolutional autoencoders. Autoencoders are generally used for image reconstruction, denoising, data dimensionality reduction and feature extraction. In this study, the convolutional autoencoder is used to extract signal features, combining both classification effect and minimum mean square error (MMSE) [?, ?] to design error function to improve the performance of feature extraction and signal reconstruction. After training the model, according to the characteristic parameter distribution of the fully connected layer, the signal of the fully connected layer is changed by occlusion and replacement to generate an interference waveform, and the BER is calculated by the receiver, the BER is used as the evaluation of the interference effect. The system model is shown in Figure 1.

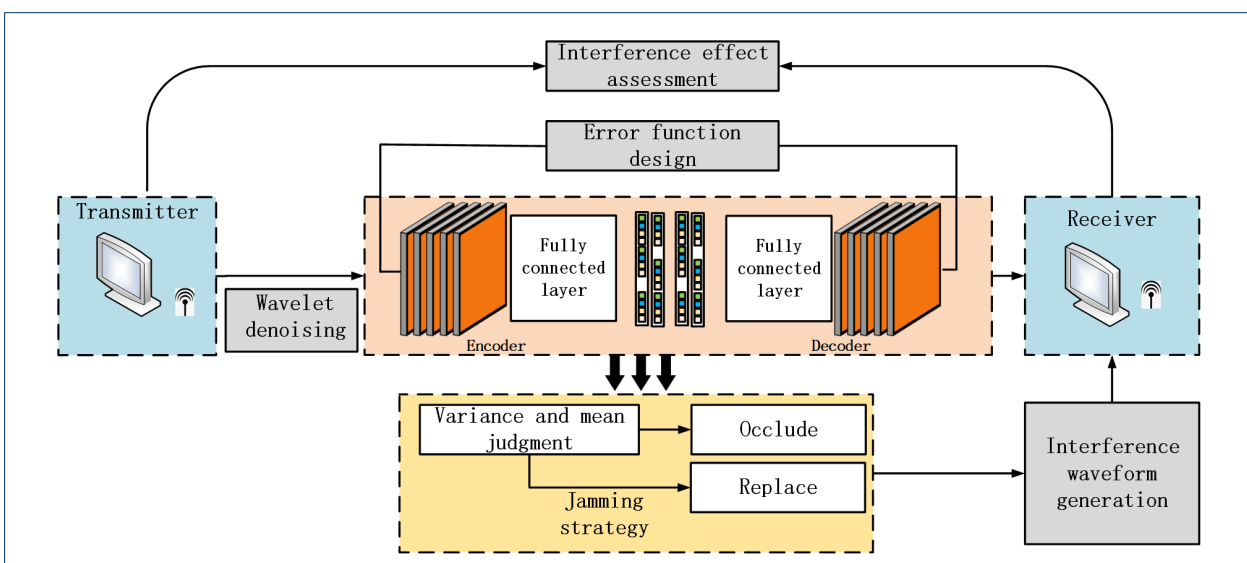

Figure 1: Interference waveform generation model

\subsubsection{Interference Waveform Generation Strategy}

According to the network structure of the convolutional autoencoder, the communication signal can be approximately reconstructed under the constraint of ensuring the classification accuracy. Therefore, it is considered that the high-dimensional features extracted by the network structure in the fully connected layer can represent the signal. In addition, literature [?, ?] have deduced the interference probability distribution functions of signals such as multiple phase shift keying (MPSK) and multiple quadrature amplitude modulation (MQAM) under specific constraints. We generate interference waveforms by destroying the characteristics of specific signals. In order to test the interference effect of interference waveforms generated by different feature perturbations, this study formulates rules for testing the significant scores of two types of signal features, which are the inter-class variance and the total mean. 
For the linear combination, the larger the mean of the signal features, the greater the impact on the follow-up. The relationship between the two can be expressed as:

$$
S_{\text {ifeature }}=\bar{x}_{i M}
$$

In the formula, $\bar{x}_{i M}$ represents the variance of the i-th feature in the feature layer extracted from the M-th signal.

If the connection between the fully connected layer and the classifier does not go through any activation function, the two are considered to be a linear combination, so the larger change in the eigenvalue is the result that obviously affects the classification. Therefore, the scoring rule using the between-class variance can be expressed as:

$$
S_{i \text { feature }}=D\left(\bar{x}_{i 1}, \bar{x}_{i 2}, \cdots, \bar{x}_{i M}\right)
$$

\subsection{Convolutional Auto-encoder}

In the field of deep learning, convolutional neural network (CNN) [?] is an algorithm model widely used by scholars. At present, various new algorithm models developed by most scholars are based on convolutional neural network. In the ideal case of not considering other problems such as fitting, the deeper the CNN layer, the more abstract the features it extracts, and the autoencoder is designed with this idea. Auto-encoder (AE) [?, ?] is a neural network that copies input to output. AE converts the input information into a high-dimensional representation of the hidden space by compressing the input information, and reconstructs the representation of the hidden space into the output.

$\mathrm{AE}$ is a neural network that copies input to output. AE converts the input information into a high-dimensional representation of the hidden space by compressing the input information, and reconstructs the representation of the hidden space into the output.

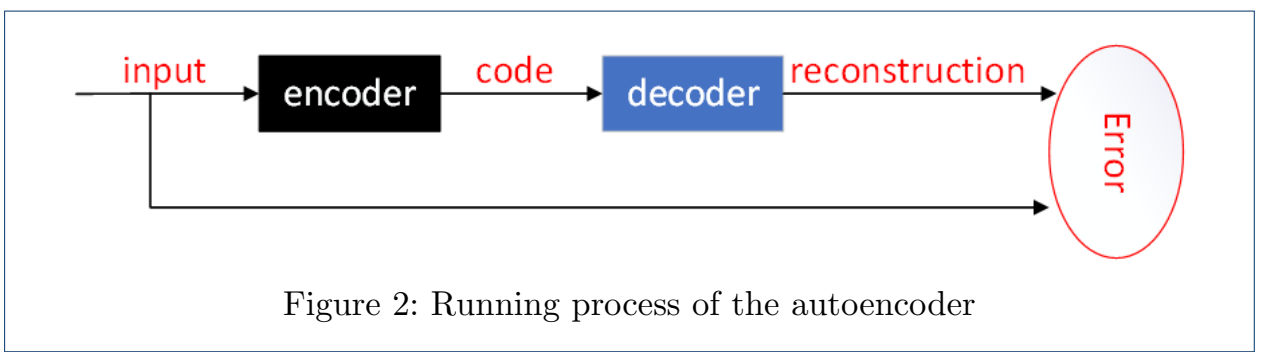

The AE operation process is shown in Figure 2. The encoder will compress the original data 'input' into 'code' and input it into the decoder, and the decoder will restore the 'code' to 'reconstruction'. Ideally, 'reconstruction' and 'input' should be exactly the same. but in practice, there must be an error between 'reconstruction' and 'input', which is also called reconstruction error in AE. Therefore, the most straightforward way to improve AE performance is to minimize the reconstruction error by adjusting the parameters of the encoder and decoder.

The basic structure of AE is generally composed of three parts: input layer, hidden layer and output layer, and its structure is shown in Figure 3. 
Assuming that the input dataset is $X=\left\{x_{1}, x_{2} \cdots, x_{N}\right\}$, the process of inputting the dataset into the encoding network can be expressed as:

$$
h_{W, b}(x)=f\left(\mathbf{W}_{\text {Encoder }} x+\mathbf{b}_{\text {Encoder }}\right)
$$

where $\mathbf{W}_{\text {Encoder }}$ and $\mathbf{b}_{\text {Encoder }}$ are the weight parameter matrix and bias matrix in the encoding network, and $f(\bullet)$ is the encoding activation function.

According to the structural symmetry of $\mathrm{AE}$, the output of the hidden layer is reconstructed as $Y=\left\{y_{1}, y_{2} \cdots, y_{N}\right\}$, where $Y=\left\{y_{1}, y_{2} \cdots, y_{N}\right\}$ is the output dataset. The process of decoding and reconstruction can be expressed as:

$$
y_{W, b}(x)=g\left(\mathbf{W}_{\text {Decoder }} h+\mathbf{b}_{\text {Decoder }}\right)
$$

where $g(\bullet)$ is the decoding activation function. It is known from the above that the most critical step to improve the performance of $\mathrm{AE}$ is to reduce the reconstruction error of $\mathrm{AE}$, so the reconstruction error $J_{A E}(\theta)$ is expressed as:

$$
J_{A E}(\theta)=\sum_{x \in \mathbf{X}} L(x, y)
$$

where $L(x, y)$ is the sample loss function:

$$
L(x, y)=\left\{\begin{array}{cc}
\|x-y\|^{2} & \mathrm{~g}(\cdot) \text { is the identity map } \\
-\left[\sum_{i=1}^{\text {Decoder }} x_{j} \log \left(y_{i}\right)+\left(1-x_{i}\right) \log \left(1-y_{i}\right)\right] & \mathrm{g}(\cdot) \text { is the sigmoid activation function }
\end{array}\right.
$$


The operation process and basic structure of AE have been introduced above. The network structure of each layer of AE mainly updates and propagates parameters through the fully connected layer. CNN is mainly a neural network composed of convolutional layers and pooling layers, in which the convolutional layer acts as a filter, and the pooling layer is responsible for extracting invariant features. The convolutional autoencoder (CAE) [?, ?, ?] replaces the fully connected layers in AE with convolutional layers and pooling layers according to the unsupervised feature extraction characteristics of CNN. The principle block diagram of CAE is shown in Figure 4.

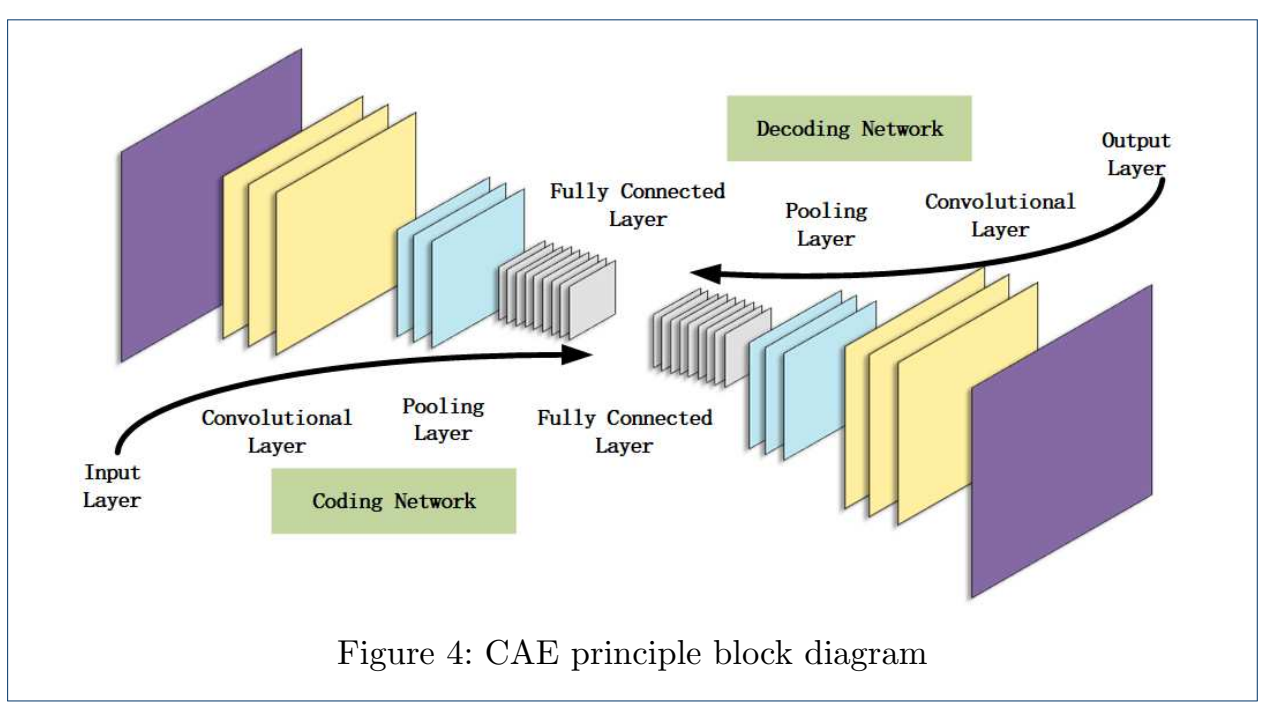

1) Convolution layer-convolution: Assuming that there are $k$ convolution kernels in CAE, each convolution kernel consists of parameters $\omega^{k}$ and $b^{k}$, firstly initialize $k$ convolution kernels, and match each convolution kernel with a bias $b$, generate $k$ features after convolving with the input, and its operation can be expressed as:

$$
h^{k}=\sigma\left(x * \omega^{k}+b^{k}\right)
$$

where $h^{k}$ is the feature map.

2) Pooling layer-pooling operation: Perform a pooling operation on the feature map $h^{k}$ generated above. The purpose of pooling is to retain the matrix of the positional relationship during pooling, which is convenient for subsequent de-pooling operations.

3) Pooling layer - self-encoding (de-pooling operation): Perform de-pooling operation on the feature map $h^{k}$ generated above, use the matrix that retains the positional relationship during pooling, and restore the data to the corresponding position of the matrix of the original size.

4) Convolutional layer - self-encoding (deconvolution operation): Each feature map $h$ and the transposition $\hat{w}$ of its corresponding convolution kernel perform a convolution operation and sum the results, and then add the bias $c$ to obtain the 
activation function $y$ :

$$
y=\sigma\left(\sum_{k} h^{k} * \hat{w}^{k}+c\right)
$$

5) Convolutional Layer - Update Weights: To update the weights, first determine the loss function. The MMSE function used here, that is, the target value minus the square sum of the actual value and then the mean value, where $2 n$ is to simplify the derivation.

$$
E=\frac{1}{2 n} \sum_{i=1}^{n}\left(x_{i}-y_{i}\right)^{2}
$$

\subsection{Loss Function}

In the encoder-based algorithm, it is generally believed that the smaller the reconstruction error, the higher the performance of the algorithm, and the error approaches zero under ideal conditions. However, Yu Z. of Southwest Jiaotong University gave the lower bound of the reconstruction error of the input data, and gave a necessary condition for the reconstruction error of the input layer to reach an ideal state. When the input data is corrupted or noise is added, the reconstruction error of corrupted or noisy input data also has a lower bound. For the loss function of the autoencoder structure, such as formula (6), the pure data input by the autoencoder network can be expressed as $x$, the input pure data can be expressed as $x_{c}^{*}$, and the abstract parameters in the implicit part of the structure can be expressed as $h_{c}$. Formula (10) can be obtained through the encoder:

$$
f(x)=h_{c}
$$

Similarly, according to its symmetric structure, the reconstructed parameters can be encoded to obtain formula (11):

$$
h_{c}^{*}=f\left(x_{c}^{*}\right)
$$

The Taylor expansion of the Lagrangian remainder for the above formula can be obtained:

$$
f\left(x_{c}^{*}\right)=f(x)+\left(x_{c}^{*}-x\right)^{T} \nabla f\left[x+\rho\left(x_{c}^{*}-x\right)\right]
$$

where $\nabla f\left[x+\rho\left(x_{c}^{*}-x\right)\right]$ is the first derivative of the coding part, where $\rho \in(0,1)$ . The loss function is represented by $h_{c}$ and $h_{c}^{*}$ as:

$$
\begin{aligned}
& L\left(h_{c}, h_{c}^{*}\right)=L\left(h_{c}, f\left(g\left(h_{c}\right)\right)\right) \\
& =\left\|h_{c}^{*}-h_{c}\right\|=\left\|f\left(x_{c}^{*}\right)-f(x)\right\|^{2}
\end{aligned}
$$

Combining (12) can get:

$$
L\left(h_{c}, h_{c}^{*}\right)=\left\|\left(x_{c}^{*}-x\right)^{T} \nabla f\left[x+\rho\left(x_{c}^{*}-x\right)\right]\right\|^{2}
$$


Let $x_{c}^{*}-x=\left|\xi_{1}, \xi_{2}, \cdots, \xi_{m}\right|^{T}$, while denoting $\nabla f\left[x+\rho\left(x_{c}^{*}-x\right)\right]$ as a real matrix $A$. Then formula (14) can be expressed as:

$$
\begin{aligned}
& L\left(h_{c}, h_{c}{ }^{*}\right)=\left\|\xi_{1}, \xi_{2}, \cdots,\left.\xi_{m}\right|^{T} \cdot A\right\|^{2}=\sum_{j=1}^{n}\left(\sum_{i=1}^{m} a_{i j} \xi_{i}\right)^{2} \\
& \leq \sum_{j=1}^{n}\left[\left(\sum_{i=1}^{m} a_{i j}^{2}\right)\left(\sum_{i=1}^{m} \xi_{i}^{2}\right)\right]=\xi^{2} \cdot A^{2}
\end{aligned}
$$

Ideally, it is hoped that the reconstructed data and the produced data are as identical as possible, which can be expressed as:

$$
\lim _{x_{c}^{*} \rightarrow x} \nabla f\left[x+\rho\left(x_{c}^{*}-x\right)\right]_{F}^{2}=J_{f}(x)_{F}^{2}
$$

Formula (17) can be obtained from the above formula:

$$
L\left(x_{c}^{*}, x\right) \geq L\left(x_{c}^{*}, h_{c}\right) /\left\|J_{f}(x)\right\|_{F}^{2}
$$

Through the network structure of the convolutional autoencoder in Section 2.4, the $1^{*} 1000$-dimensional features of the signal are connected to the classifier through the encoded information of the fully connected layer. The confidence loss for classification represented by the Softmax [?, ?] function is shown in formula (18):

$$
L_{\text {cross }}=L_{\text {Soft }}-\frac{1}{N} \sum_{N}^{i=1} \log \left(\frac{e^{h_{y_{i}}}}{\sum_{C} e^{h_{j}}}\right)
$$

The classification accuracy of the signal is evaluated by the normal cross-entropy loss. When this part of the network can complete the classification of the signal, it can be considered that the network has extracted significant information in each category, so the deconvolution network can be used to reconstruct the signal on this basis. In the previous coding structure, the position of the reserved value in each operation in the pooling layer is recorded, and the deconvolutional signal is expanded to the same dimension as the original according to the above position during deconvolution. The data format of the final structure output is $2 * 1024$. According to the above derivation, the sum of the reconstruction error and the classification error is guaranteed to be the smallest, so the error formula is obtained as follows:

$$
L_{\text {mypaper }}=\alpha L_{\text {classify }}+\|x-y\|^{2}-L\left(x_{c}^{*}, h_{c}\right) /\left\|J_{f}(x)\right\|_{F}^{2}
$$

where $L_{\text {classify }}$ is the Softmax cross-entropy loss above, and $\alpha$ is a hyperparameter. To balance the magnitude relationship between the two and accelerate the training convergence, this study sets this parameter to 0.1 .

\subsection{Network Structure}

According to the characteristics of the interference signal, it is considered that the interference signal needs to overlap with the communication signal in the timefrequency domain, and should have similar characteristics in the communication 
signal. Therefore, a convolutional encoder and a classification loss structure are proposed in this to generate interference signals, and the structure box is shown in Figure. 5.

Table 1: Encoding part of the network structure

\begin{tabular}{|c|c|c|}
\hline Network Structure & Convolution Kernel Structure & Output Layer \\
\hline Input & 7 & $(2,1024)$ \\
\hline Convolutional layer (normalization) & $(2,2,128)$ & l \\
\hline ReLU/PReLU & 128 & / \\
\hline Convolutional layer (normalization) & $(1,2,128)$ & / \\
\hline ReLU/PReLU & 128 & / \\
\hline Pooling layer (max pooling) & $(1,2,128)$ stride $=2$ & / \\
\hline Convolutional layer (normalization) & $(1,2,256)$ & / \\
\hline ReLU/PReLU & 256 & / \\
\hline Convolutional layer (normalization) & $(1,2,256)$ & / \\
\hline ReLU/PReLU & 256 & / \\
\hline Pooling layer (max pooling) & $(1,2,256)$ stride $=2$ & / \\
\hline Convolutional layer (normalization) & $(1,2,512)$ & / \\
\hline ReLU/PReLU & 512 & / \\
\hline Convolutional layer (normalization) & $(1,2,512)$ & / \\
\hline ReLU/PReLU & 512 & / \\
\hline Pooling layer (max pooling) & $(1,2,512)$ stride $=2$ & / \\
\hline Fully connected layer (dropout $=0.5$ ) & $256 * 128 * 1$ & $1000 * 1$ \\
\hline Fully connected layer (dropout $=0.5$ ) & $1000 * 1$ & $1000 * 1$ \\
\hline Classifier & $1000 * 3$ & $3 * 1$ \\
\hline
\end{tabular}

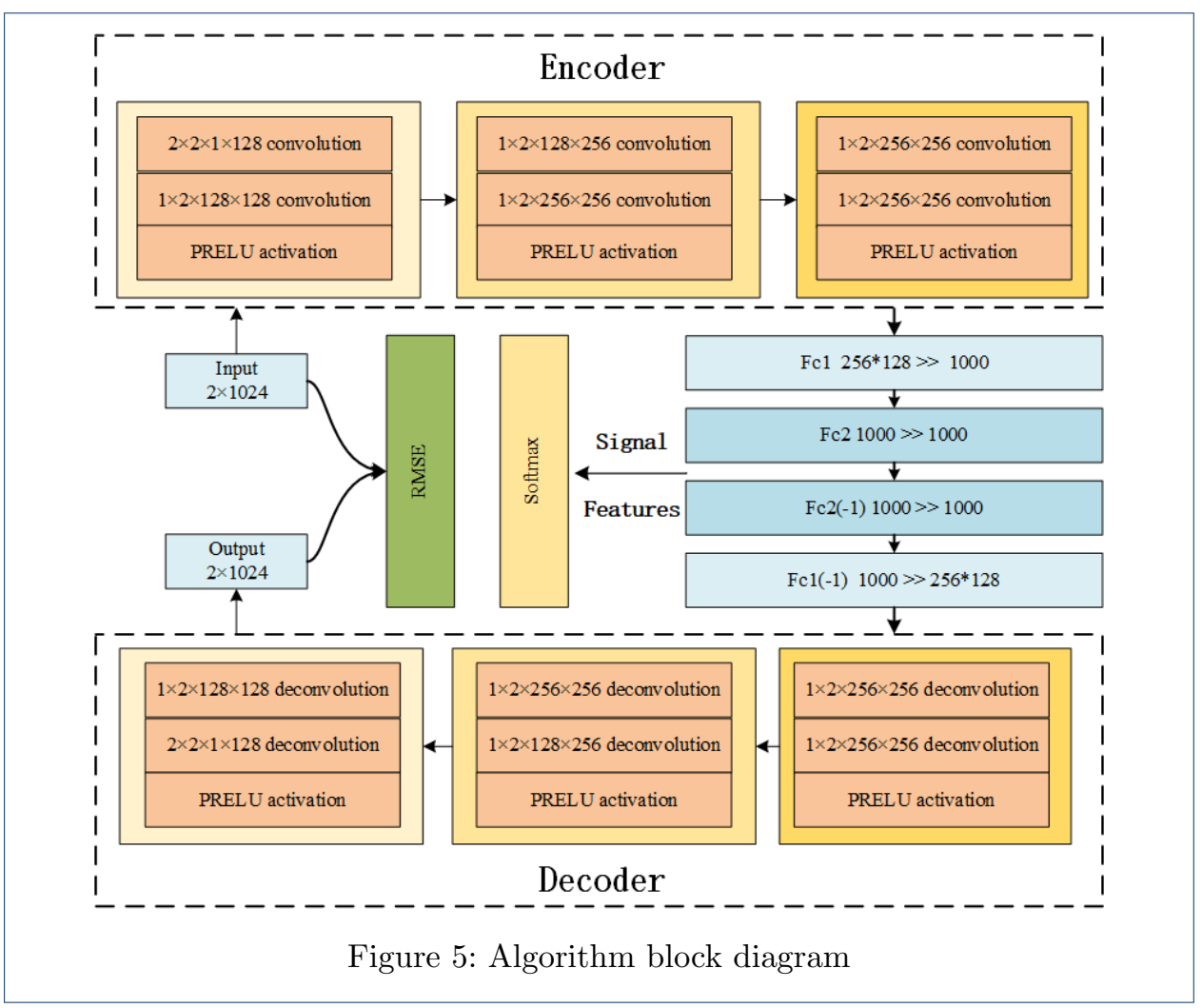

Through the convolutional neural network in the above table, the signal is converted into features of dimension $1^{*} 1000$. For a signal with a length of $2^{*} 1024$, only a fully connected layer with a smaller dimension is required to describe the features. The purpose of using a longer dimension in this study is to provide a larger policy 
dimension space for subsequent generation of interference waveforms. The classifier is connected through the encoded information of the fully connected layer, and the upper part of the encoder is connected to the Softmax classifier, so that the neural network can extract the salient features of the communication signal in this way. At the same time, the fully connected layer is connected to the deconvolution network for decoding the communication signal. According to the symmetry of the structure of the autoencoder and Table 1, the structure of the decoder can be reversely restored.

\section{Results and discussion}

To test the feasibility of the interference waveform generation technology proposed in this study, we divide the experiment into pattern analysis of generated signal and interference effect comparison experiment.

\subsection{Analysis of Algorithm Reconstruction Results}

To verify the subsequent interference effect, the reconstruction performance of the convolutional autoencoder needs to be tested first. In this experiment, the features with significant scores are kept unchanged while other non-salient features are changed. The $1 * 1000$-dimensional features are distributed in a ratio of 10:990, and the non-salient features or salient features are replaced with Gaussian random noise with the same energy to reconstruct the signal. The experimental results are illustrated in Figures 6 and 7.
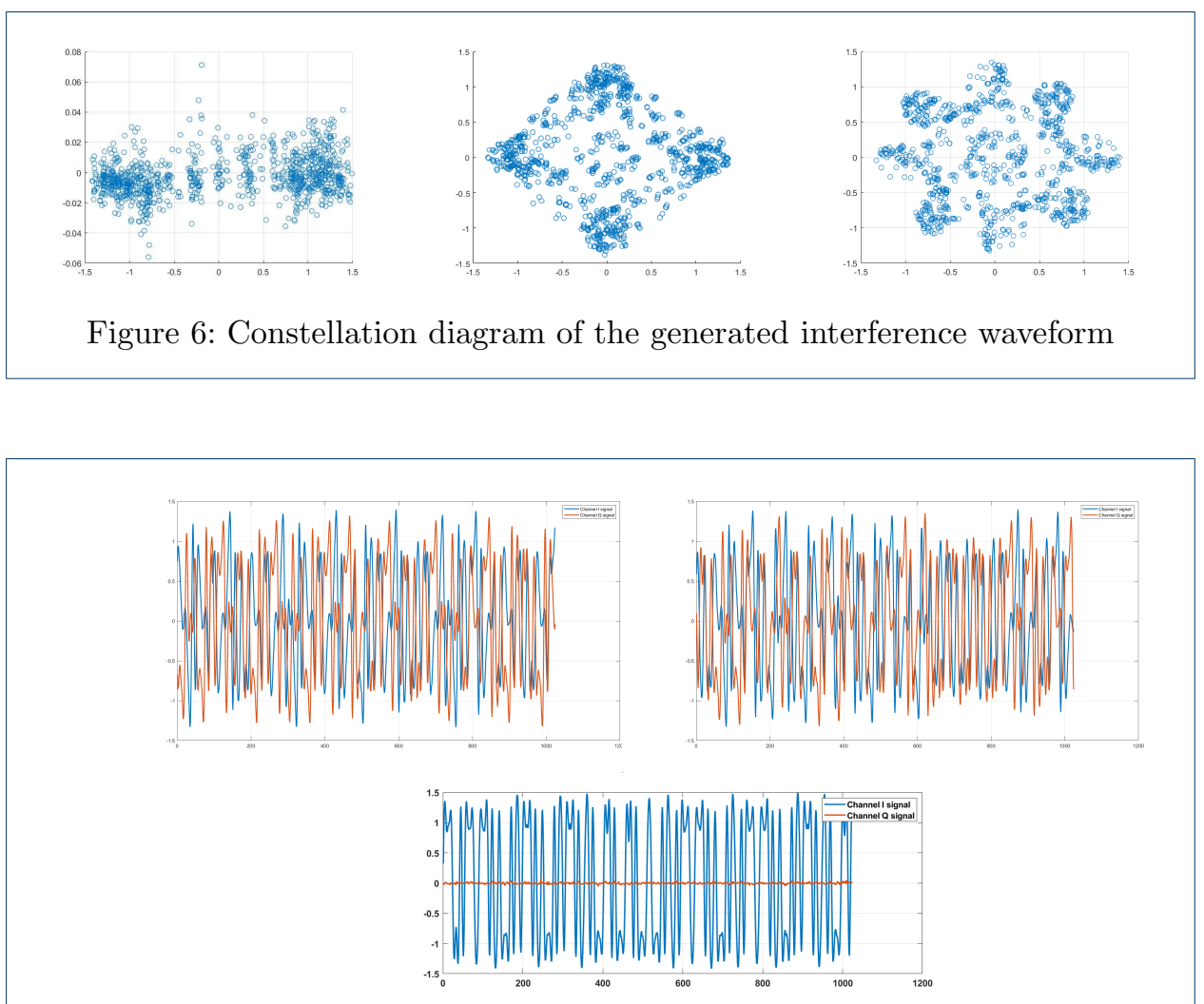

Figure 7: Randomly generated interference waveform 
To detect the similarity between the interference signal and the communication signal more intuitively, the above figures illustrate the time domain I/Q waveform and constellation diagram of the generated interference.

The BSPK, QPSK, and 8PSK signals can be clearly distinguished from the constellation diagram, and the time-domain curve of the communication signal is smooth, which proves that the reconstructed interference waveform has a strong similarity with the communication waveform. However, there is a large gap between the constellation diagram of the BPSK signal and the original signal constellation diagram. This is because the unified mean square error is used in the training process, and the amplitude difference between the Q-channel signal of the BPSK signal and the other two types is too large.

\subsection{Analysis of the interference results of the algorithm}

This experiment adds perturbation and occlusion to the features in the fully connected layer, and verifies the interference performance of the waveform generated by the deconvolution. In order to compare and test the interference effect, the SNR is selected as $15 \mathrm{~dB}$, the communication mode is BPSK, QPSK, 8PSK, the SIR is $-1510 \mathrm{~dB}$, the step is $2 \mathrm{~dB}$, and a random string with a length of 1200 is used. The ASCII source code is used as a test sequence, and 100 tests are performed and the average value of the BER is taken. Select BPSK, QPSK and 8PSK three kinds of communication signal itself, noise amplitude modulation, Gaussian noise and other interference for comparison, and the interference effect curve is shown in Figure. 8.

In this experiment, the maximum weight of $1 \%$ of the fully connected layer is selected to apply Gaussian perturbation according to the two standards of mean and standard deviation, and the mean value of the fully connected layer is kept unchanged. The output interference signals are deconvolution interference waveform 1 and deconvolution interference waveform 2 respectively. The low interference performance in BPSK communication is because a certain amount of energy is allocated to the Q-channel signal in the waveform reconstruction, resulting in the degradation of the interference performance. However, the BER of QPSK and 8PSK communication waveforms are both higher than noise amplitude modulation and Gaussian noise interference.

In summary, the method proposed in this study can generate an interference waveform that approximates the communication signal, and is better than the interference performance of Gaussian noise and noise amplitude modulation under different SIRs.

\section{Conclusion}

Aiming at the problem of signal management and control in the IoV, this study introduces the intelligent interference of communication signals into the signal processing of the IoV, and proposes a method for generating interference waveforms based on convolutional autoencoders. To ensure the applicability and validity of the experimental results, a complete vehicle network communication system is simulated and built in this study, and three communication signals of BPSK, QPSK and 8PSK are used for communication. In this study, a method based on convolutional autoencoder is proposed to extract high-order features of communication 

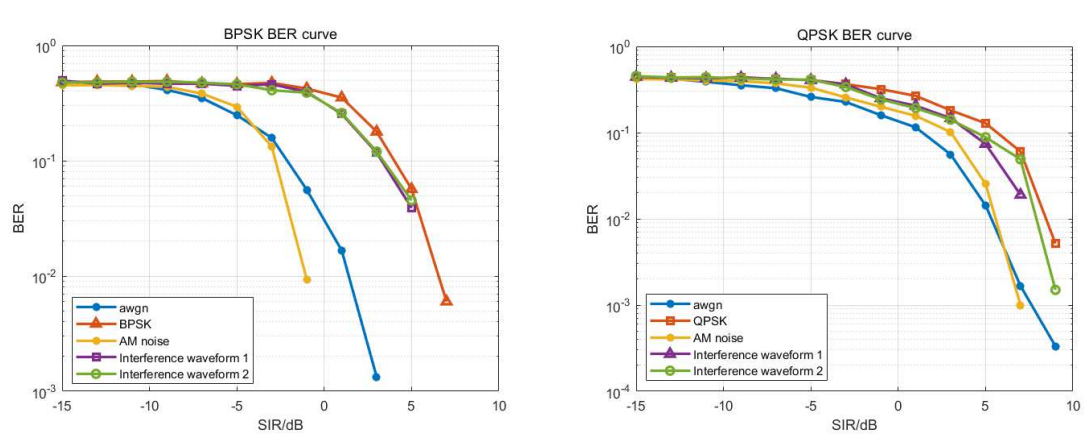

(a) BER Curve of BPSK Communica- (b) BER Curve of QPSK Communication System tion System

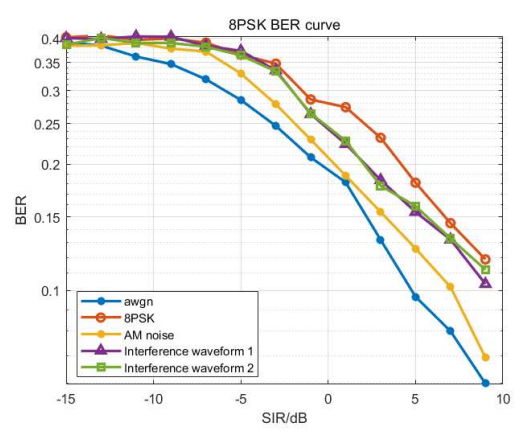

(c) BER Curve of 8PSK Communication System

Figure 8: BER curve under the generated interference waveform $(\mathrm{SNR}=15 \mathrm{~dB})$ 
signals, and the required intelligent interference waveform is obtained by occluding and replacing the features on the fully connected layer. It can be seen from the simulated constellation diagram that the reconstructed interference waveform has a strong similarity with the communication waveform, which proves the similarity between the generated interference waveform and the original signal waveform. In addition, the bit error rate curve obtained by simulation can prove that the interference performance of the interference waveform generated in this study is better than that of Gaussian noise and noise amplitude modulation under different SIRs.

The modulation methods applied to the IoV signals are complex and diverse, and this study only focuses on three phase shift keying signals for communication system construction and intelligent interference. Therefore, in future research, the types of modulation methods included should be expanded, so as to achieve comprehensive control of IoV signals.

\begin{abstract}
Abbreviations
IoV: Internet of Vehicles; BER: Bit error rate; SIR: Signal-to-interference ratio; SARSA:

State-action-reward-state-action; Fill-CFAR:Fill collaborative forecast and replenishment; BPSK: Binary phase shift keying; QPSK: Quaternary phase shift keying; 8PSK: Octal phase shift keying; SNR: Signal-to-noise ratio; Am: Amplitude modulation; MMSE: Minimum mean square error; MPSK: Multiple phase shift keying; MQAM: Multiple quadrature amplitude modulation; CNN: Convolutional neural network; AE: Auto-encoder; CAE: Convolutional auto-encoder.
\end{abstract}

\title{
Acknowledgements
}

Not applicable.

\section{Authors' contributions}

$\mathrm{CY}$ proposed the framework of the whole algorithm; $\mathrm{XY}$ performed the simulations, analysis and interpretation of the results. CB and ZA have participated in the conception and design of this research, and revised the manuscript. All authors read and approved the fnal manuscript.

\section{Funding}

This work is supported by the National Natural Science Foundation of China (62001137); the Natural Science Foundation of Heilongjiang Province (JJ2019LH2398); the Fundamental Research Funds for the Central Universities (3072021CF0805).

\section{Availability of data and materials}

Please contact author for data requests.

Ethics approval and consent to participate

Not applicable.

\section{Consent for publication}

The picture materials quoted in this article have no copyright requirements, and the source has been indicated.

Competing interests

The authors declare that they have no competing interests.

Author details

?? College of Information and Communication Engineering, Harbin Engineering University, Harbin, China. ?? Key Laboratory of Advanced Marine Communication and Information Technology, Ministry of Industry and Information Technology, Harbin Engineering University, Harbin, China.

\section{References}

1. Fu, E., Li, X., Yao, Z., Ren, Y., Wu, Y., Fan, Q.: Personnel emotion recognition model for internet of vehicles security monitoring in community public space. EURASIP Journal on Advances in Signal Processing 2021(1), 1-19 (2021)

2. Yazdi, H.S., Lotfizad, M., Kabir, E., Fathy, M.: Clipped input rls applied to vehicle tracking. EURASIP Journal on Advances in Signal Processing 2005(8), 1-8 (2005)

3. Liu, X., Zhang, X.: Noma-based resource allocation for cluster-based cognitive industrial internet of things. IEEE transactions on industrial informatics 16(8), 5379-5388 (2019)

4. Liu, X., Zhai, X.B., Lu, W., Wu, C.: Qos-guarantee resource allocation for multibeam satellite industrial internet of things with noma. IEEE Transactions on Industrial Informatics 17(3), 2052-2061 (2019)

5. Wei, B., Zhang, L., Wang, K., Kong, Q., Wang, Z.: Dynamic scene deblurring and image de-raining based on generative adversarial networks and transfer learning for internet of vehicle. EURASIP Journal on Advances in Signal Processing 2021(1), 1-19 (2021) 
6. Arróspide, J., Salgado, L., Nieto, M.: Video analysis-based vehicle detection and tracking using an $\mathrm{mcmc}$ sampling framework. EURASIP Journal on Advances in Signal Processing 2012(1), 1-20 (2012)

7. Xu, X., Wang, Y., Liu, J., Zhang, X.: Analysis on the achievement milestones and limitations of game theory. In: 2008 Chinese Control and Decision Conference, pp. 1214-1219 (2008). IEEE

8. Shafiee, S., Ulukus, S.: Capacity of multiple access channels with correlated jamming. In: Military Communications Conference, 2005. MILCOM 2005. IEEE (2005)

9. Kashyap, A., Basar, T., Srikant, R.: Correlated jamming on mimo gaussian fading channels. IEEE Transactions on Information Theory (2006)

10. Chang, L.: An information-theoretic study of ratio-threshold antijam techniques. thesis illinois univ (1985)

11. Amuru, S., Buehrer, R.: Optimal jamming against digital modulation. IEEE Transactions on Information Forensics \& Security 10(10), 2212-2224 (2015)

12. Farzamnia, A., Hlaing, N.W., Haldar, M.K., Rahebi, J.: Channel estimation for sparse channel ofdm systems using least square and minimum mean square error techniques. In: 2017 International Conference on Engineering and Technology (ICET) (2018)

13. Yang, C., Yang, F., Wang, Z.: Iterative minimum mean square error equalization for optical fiber communication systems. IEEE Photonics Technology Letters 19(19), 1571-1573 (2007)

14. Huang, L., Neely, M.J.: The optimality of two prices: Maximizing revenue in a stochastic communication system. IEEE/ACM transactions on networking 18(2), 406-419 (2009)

15. Uçkun, F., Özer, H., Nurbaș, E., Onat, E.: Direction finding using convolutional neural networks and convolutional recurrent neural networks. In: 2020 28th Signal Processing and Communications Applications Conference (SIU), pp. 1-4 (2020)

16. Zhou, Y., Amimeur, A., Jiang, C., Dou, D., Jin, R., Wang, P.: Density-aware local siamese autoencoder network embedding with autoencoder graph clustering. In: 2018 IEEE International Conference on Big Data (Big Data), pp. 1162-1167 (2018). IEEE

17. Xu, Q., Wu, Z., Yang, Y., Zhang, L.: The difference learning of hidden layer between autoencoder and variational autoencoder. In: 2017 29th Chinese Control And Decision Conference (CCDC), pp. 4801-4804 (2017). IEEE

18. Zhao, X., Han, X., Su, W., Yan, Z.: Time series prediction method based on convolutional autoencoder and Istm. In: 2019 Chinese Automation Congress (CAC), pp. 5790-5793 (2019). IEEE

19. Chun, C., Jeon, K.M., Kim, T., Choi, W.: Drone noise reduction using deep convolutional autoencoder for uav acoustic sensor networks. In: 2019 IEEE 16th International Conference on Mobile Ad Hoc and Sensor Systems Workshops (MASSW) (2019)

20. Lee, H., Kim, J., Kim, B., Kim, S.: Convolutional autoencoder based feature extraction in radar data analysis. In: 2018 Joint 10th International Conference on Soft Computing and Intelligent Systems (SCIS) and 19th International Symposium on Advanced Intelligent Systems (ISIS), pp. 81-84 (2018). IEEE

21. Rao, Q., Yu, B., He, K., Feng, B.: Regularization and iterative initialization of softmax for fast training of convolutional neural networks. In: 2019 International Joint Conference on Neural Networks (IJCNN), pp. 1-8 (2019). IEEE

22. Hussain, M.A., Tsai, T.-H.: An efficient and fast softmax hardware architecture (efsha) for deep neural networks. In: 2021 IEEE 3rd International Conference on Artificial Intelligence Circuits and Systems (AICAS), pp. 1-4 (2021). IEEE 


\title{
RESEARCH
}

\section{Signal Processing of Internet of Vehicles Based on Intelligent Interference}

\author{
Xiangyu $\mathrm{Wu}^{1}$, Changbo Hou ${ }^{1,2}$, Zhian Deng ${ }^{1,2^{*}}$, Chenyu Fang ${ }^{1}$ and Guowei Liu ${ }^{1}$
}

${ }^{*}$ Correspondence:

dengzhian@hrbeu.edu.cn

${ }^{1}$ College of Information and Communication Engineering, Harbin Engineering University, Harbin, China

Full list of author information is available at the end of the article

\begin{abstract}
With the rapid development of in-vehicle electronic technology and artificial intelligence, Internet of Vehicles (loV) technology, as an effective integration of the two, greatly reduces the probability of road traffic accidents. However, the current loV system is not perfect for the control process of abnormal vehicles. Therefore, in order to strengthen the management and control of abnormal vehicles in the loV, it is extremely necessary to propose a method for interfering with loV signals. Among the current popular intelligent interference methods, most of them rely on the prior knowledge of the signal to deduce the best interference waveform. However, These methods rely too much on prior knowledge to be practically applicable. Therefore, in view of the shortcomings of the current communication interference technology, this study proposes an interference waveform generation technology based on convolutional autoencoders. The convolutional autoencoder was used to change the features on the fully connected layer to generate an interference waveform that is very similar to the received signal waveform, and the interference waveform is sent to the receiver to realize the control of the loV signal. The simulation results show that the interference waveform generation technology proposed in this study can make the bit error rate (BER) reach $38.4 \%$ within the signal-to-interference ratio (SIR) from $-10 \mathrm{~dB}$ to $-15 \mathrm{~dB}$.
\end{abstract}

Keywords: loV signal processing; Intelligent interference; Convolutional autoencoder; Interference waveform generation

\section{Introduction}

With the development of artificial intelligence technology, in the future $6 \mathrm{G}$ era, various network models of deep learning will be ubiquitous. One of the most critical scenarios is that service providers need to interact and make decisions on vehicle information in real time $[1,2]$.

IoV is based on the Internet of Things $[3,4]$ technology and is more oriented to transportation applications.At present, the IoV can obtain environmental information around the vehicle through the image sensor on the vehicle $[5,6]$. This information processing technology has greatly improved traffic safety. However, the current signal processing technology of the IoV is not perfect. When the owner deliberately violates the rules or the vehicle sensing system fails, it is difficult to avoid traffic accidents only by relying on the subjective initiative of the owner. Therefore, safe, effective and intelligent interference to illegal driving behavior is an important field of IoV signal control. IoV signal control is based on IoV signal processing. Therefore, to improve the abnormal IoV signal control technology, this study introduces intelligent interference technology into the field of IoV signal processing. 
At present, interference methods for digital communication are still dominated by traditional interference methods, which hinder the target receiver from receiving information through high-power suppression. However, the density of traffic flow on urban roads is relatively large, and the number of vehicles with abnormal conditions is often a small number. High-power barrage jamming cannot distinguish specific interference targets, which means that traditional interference methods are not suitable for IoV signal processing. Therefore, the introduction of intelligent communication interference into IoV signal processing has become a major trend in the development of the current IoV field.

Research on intelligent interference has been carried out for several years at home and abroad. Traditional methods are generally based on optimization theory, information theory, game theory [7] and other technologies. They rely on prior knowledge and combine signal analysis optimization and game theory to obtain optimal interference strategies and optimal interference waveforms under specific constraints. References $[8,9,10]$ analyze the capacity of wireless channels in the presence of correlated interference, and prove that Gaussian signaling and Gaussian interference form a saddle-point solution. In 2016, Scholars such as Amuru deduced the theoretical optimal interference waveforms for several classical digital modulations, and proved that it is not optimal to match the jammer's signal with the disturbed signal to obtain the maximum BER probability. These interference methods based on optimization theory need to have certain prior knowledge of the modulation method of the disturbed signal and the communication network environment, so that the corresponding optimal interference waveform can be accurately analyzed. When the environment changes, the algorithm often cannot adapt quickly according to the parameter changes of the target.

Thanks to the rapid development of computer equipment, scholars have begun to study the interference algorithm of artificial intelligence. The work done at home and abroad mainly focuses on artificial intelligence algorithms, makes autonomous decisions in terms of interference strategies, and finds the best interference pattern under the existing interference waveform pattern library. Amuru et al. [11] proposed to use a multi-armed bandit machine to model the interference waveform decision problem, and studied the interference strategies for one-to-one and many-to-many scenarios in the absence of prior information. At the same time, continuous and discrete action state spaces are established, which provides a reference for subsequent modeling and exploration based on reinforcement learning methods. Yang H.J. et al. applied the reinforcement learning algorithm to the selection of the interference channel, and explored the action space based on the power and signal modulation method through the reinforcement learning network, so as to achieve the purpose of tracking the dynamic interference of the enemy target. Zhang J.Y. searched the entire strategy combination space by means of Gaussian perturbation exploration, generates the optimal interference strategy, and improved the selection speed of the optimal interference strategy. Wu Z.G. et al. proposed reinforcement learning methods based on state-action-reward-state-action (SARSA) and Q-learning, optimized the design of interference waveforms, and verified the feasibility of the fill collaborative forecast and replenishment (Fill-CFAR) method of interference design. Zhang X.M. et al. established a Markov chain dynamic interference model and used 
reinforcement learning to design interference waveforms with various exploration strategies.

However, these methods above rely too much on prior information, and in practical application scenarios, it is very difficult to obtain prior information of the disturbed signal.

Therefore, for the case of unknown prior information, this study proposes a method based on convolutional autoencoder to parse the received signal and generate an interference waveform with similar characteristics to the original signal. In this study, we choose to interfere with the communication signals of three modulation methods: binary phase shift keying (BPSK), quaternary phase shift keying (QPSK) and octal phase shift keying (8PSK) . After denoising, the signal sent by the transmitter is input into the convolutional auto-encoder to extract signal features, and the interference waveform is generated by changing the parameters of the fully connected layer, and the interference effect is evaluated at the receiver. This study makes the following three contributions to the problem of signal management and control of the IoV:

1) The method used in this study does not rely on prior knowledge.

2) The shape of the interference waveform generated by the method used in this study is very similar to the shape of the disturbed signal waveform, so that the receiver cannot judge whether the interference is applied or not.

3) The interference effect of the method adopted in this study is superior to Gaussian noise interference and amplitude modulation (AM) noise interference under any SIR. When the signal-to-noise ratio(SNR) is $15 \mathrm{~dB}$, and the SIR is within $-10 \mathrm{~dB}$ to $-15 \mathrm{~dB}$, the interference waveform generation technology proposed in this study can make the bit error rate reach $38.4 \%$.

This study is organized as follows: Section 2 presents the method used in this study, and describes the model and algorithm network structure of the optimal interference waveform generation based on convolutional autoencoders proposed in this study. Section 3 presents the simulation results of the experimental simulation. Section 4 draws conclusions and summarizes future research directions.

\section{Methods}

\subsection{System model}

Traditional interference generally selects the interference waveform that best matches the characteristics of the received signal from the strategy library to impose interference. However, for the signal of the unknown system, it is impossible to find a suitable interference method, and generally barrage interference is used to interfere with the other party. However, the barrage interference has the problem that the required power is too large and cannot be accurately interfered. Therefore, this study proposes a structure based on the convolutional autoencoder to extract features from the signal, and adds perturbations to the features of the fully connected layer to generate interference signals with similar high-level abstract features to the target signal.

\subsubsection{Model of Interference Waveform Generation Based on Autoencoder}

When the modulation mode adopted by the communication signal is known, the probability density function of its efficient interference can be derived by mathematical formula, and the interference signal can be generated according to the 
distribution characteristics. However, in the absence of prior knowledge of communication signals, signals with similar characteristics in time domain, frequency domain, information domain, energy domain and feature domain can still be considered as efficient interference. Therefore, this proposes a feature extraction method based on convolutional autoencoders. Autoencoders are generally used for image reconstruction, denoising, data dimensionality reduction and feature extraction. In this study, the convolutional autoencoder is used to extract signal features, combining both classification effect and minimum mean square error (MMSE) $[12,13]$ to design error function to improve the performance of feature extraction and signal reconstruction. After training the model, according to the characteristic parameter distribution of the fully connected layer, the signal of the fully connected layer is changed by occlusion and replacement to generate an interference waveform, and the BER is calculated by the receiver, the BER is used as the evaluation of the interference effect. The system model is shown in Figure 1.

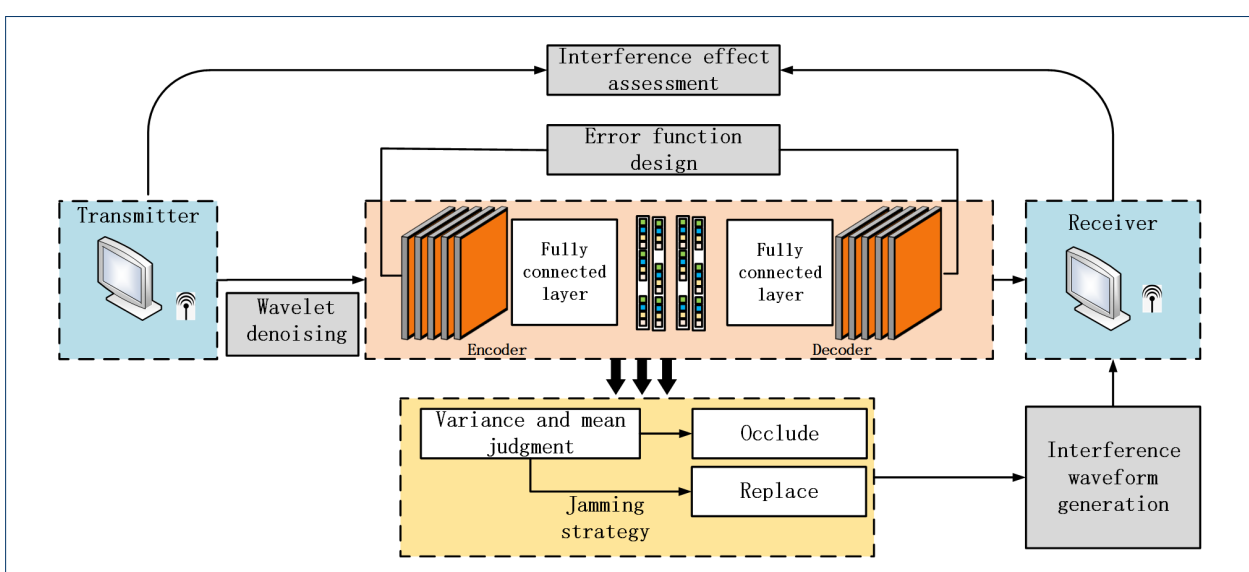

Figure 1: Interference waveform generation model

\subsubsection{Interference Waveform Generation Strategy}

According to the network structure of the convolutional autoencoder, the communication signal can be approximately reconstructed under the constraint of ensuring the classification accuracy. Therefore, it is considered that the high-dimensional features extracted by the network structure in the fully connected layer can represent the signal. In addition, literature $[11,14]$ have deduced the interference probability distribution functions of signals such as multiple phase shift keying (MPSK) and multiple quadrature amplitude modulation (MQAM) under specific constraints. We generate interference waveforms by destroying the characteristics of specific signals. In order to test the interference effect of interference waveforms generated by different feature perturbations, this study formulates rules for testing the significant scores of two types of signal features, which are the inter-class variance and the total mean.

For the linear combination, the larger the mean of the signal features, the greater the impact on the follow-up. The relationship between the two can be expressed as:

$$
S_{\text {ifeature }}=\bar{x}_{i M}
$$


In the formula, $\bar{x}_{i M}$ represents the variance of the i-th feature in the feature layer extracted from the M-th signal.

If the connection between the fully connected layer and the classifier does not go through any activation function, the two are considered to be a linear combination, so the larger change in the eigenvalue is the result that obviously affects the classification. Therefore, the scoring rule using the between-class variance can be expressed as:

$$
S_{\text {ifeature }}=D\left(\bar{x}_{i 1}, \bar{x}_{i 2}, \cdots, \bar{x}_{i M}\right)
$$

\subsection{Convolutional Auto-encoder}

In the field of deep learning, convolutional neural network (CNN) [15] is an algorithm model widely used by scholars. At present, various new algorithm models developed by most scholars are based on convolutional neural network. In the ideal case of not considering other problems such as fitting, the deeper the CNN layer, the more abstract the features it extracts, and the autoencoder is designed with this idea. Auto-encoder (AE) $[16,17]$ is a neural network that copies input to output. AE converts the input information into a high-dimensional representation of the hidden space by compressing the input information, and reconstructs the representation of the hidden space into the output.

$\mathrm{AE}$ is a neural network that copies input to output. AE converts the input information into a high-dimensional representation of the hidden space by compressing the input information, and reconstructs the representation of the hidden space into the output.

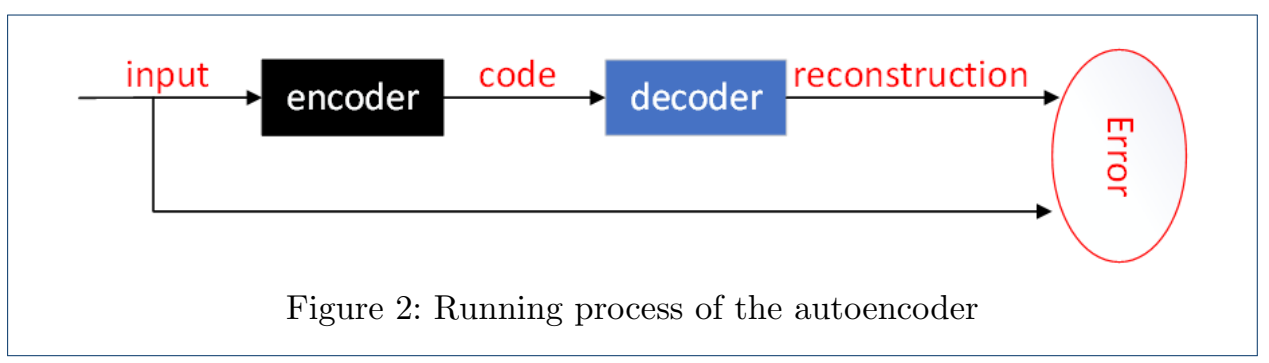

The AE operation process is shown in Figure 2. The encoder will compress the original data 'input' into 'code' and input it into the decoder, and the decoder will restore the 'code' to 'reconstruction'. Ideally, 'reconstruction' and 'input' should be exactly the same. but in practice, there must be an error between 'reconstruction' and 'input', which is also called reconstruction error in AE. Therefore, the most straightforward way to improve $\mathrm{AE}$ performance is to minimize the reconstruction error by adjusting the parameters of the encoder and decoder.

The basic structure of AE is generally composed of three parts: input layer, hidden layer and output layer, and its structure is shown in Figure 3.

Assuming that the input dataset is $X=\left\{x_{1}, x_{2} \cdots, x_{N}\right\}$, the process of inputting the dataset into the encoding network can be expressed as:

$$
h_{W, b}(x)=f\left(\mathbf{W}_{\text {Encoder }} x+\mathbf{b}_{\text {Encoder }}\right)
$$


where $\mathbf{W}_{\text {Encoder }}$ and $\mathbf{b}_{\text {Encoder }}$ are the weight parameter matrix and bias matrix in the encoding network, and $f(\bullet)$ is the encoding activation function.

According to the structural symmetry of AE, the output of the hidden layer is reconstructed as $Y=\left\{y_{1}, y_{2} \cdots, y_{N}\right\}$, where $Y=\left\{y_{1}, y_{2} \cdots, y_{N}\right\}$ is the output dataset. The process of decoding and reconstruction can be expressed as:

$$
y_{W, b}(x)=g\left(\mathbf{W}_{\text {Decoder }} h+\mathbf{b}_{\text {Decoder }}\right)
$$

where $g(\bullet)$ is the decoding activation function. It is known from the above that the most critical step to improve the performance of $\mathrm{AE}$ is to reduce the reconstruction error of AE, so the reconstruction error $J_{A E}(\theta)$ is expressed as:

$$
J_{A E}(\theta)=\sum_{x \in \mathbf{X}} L(x, y)
$$

where $L(x, y)$ is the sample loss function:

$L(x, y)=\left\{\begin{array}{cc}\|x-y\|^{2} & \mathrm{~g}(\cdot) \text { is the identity map } \\ -\left[\sum_{i=1}^{\text {Decoder }} x_{j} \log \left(y_{i}\right)+\left(1-x_{i}\right) \log \left(1-y_{i}\right)\right] & \mathrm{g}(\cdot) \text { is the sigmoid activation function }\end{array}\right.$

The operation process and basic structure of AE have been introduced above. The network structure of each layer of AE mainly updates and propagates parameters through the fully connected layer. CNN is mainly a neural network composed of 
convolutional layers and pooling layers, in which the convolutional layer acts as a filter, and the pooling layer is responsible for extracting invariant features. The convolutional autoencoder (CAE) [18, 19, 20] replaces the fully connected layers in $\mathrm{AE}$ with convolutional layers and pooling layers according to the unsupervised feature extraction characteristics of CNN. The principle block diagram of CAE is shown in Figure 4.

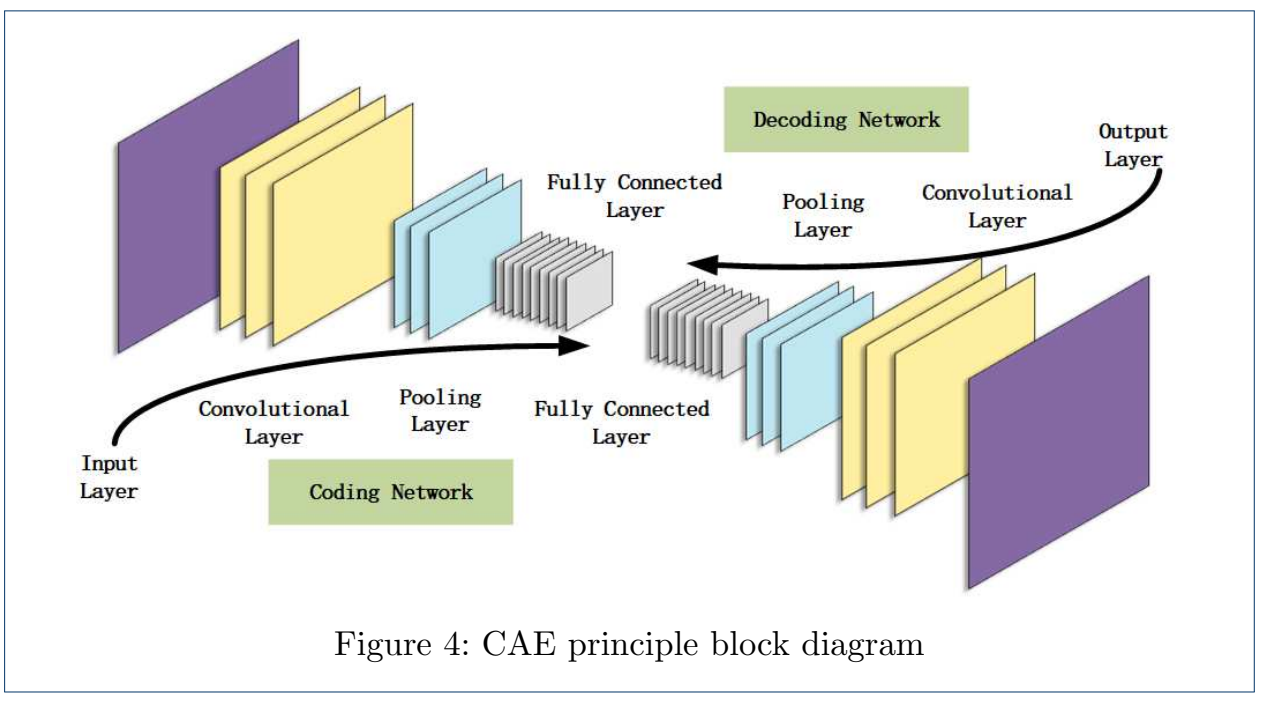

1) Convolution layer-convolution: Assuming that there are $k$ convolution kernels in CAE, each convolution kernel consists of parameters $\omega^{k}$ and $b^{k}$, firstly initialize $k$ convolution kernels, and match each convolution kernel with a bias $b$, generate $k$ features after convolving with the input, and its operation can be expressed as:

$$
h^{k}=\sigma\left(x * \omega^{k}+b^{k}\right)
$$

where $h^{k}$ is the feature map.

2) Pooling layer-pooling operation: Perform a pooling operation on the feature map $h^{k}$ generated above. The purpose of pooling is to retain the matrix of the positional relationship during pooling, which is convenient for subsequent de-pooling operations.

3) Pooling layer - self-encoding (de-pooling operation): Perform de-pooling operation on the feature map $h^{k}$ generated above, use the matrix that retains the positional relationship during pooling, and restore the data to the corresponding position of the matrix of the original size.

4) Convolutional layer - self-encoding (deconvolution operation): Each feature map $h$ and the transposition $\hat{w}$ of its corresponding convolution kernel perform a convolution operation and sum the results, and then add the bias $c$ to obtain the activation function $y$ :

$$
y=\sigma\left(\sum_{k} h^{k} * \hat{w}^{k}+c\right)
$$

5) Convolutional Layer - Update Weights: To update the weights, first determine the loss function. The MMSE function used here, that is, the target value minus 
the square sum of the actual value and then the mean value, where $2 n$ is to simplify the derivation.

$$
E=\frac{1}{2 n} \sum_{i=1}^{n}\left(x_{i}-y_{i}\right)^{2}
$$

\subsection{Loss Function}

In the encoder-based algorithm, it is generally believed that the smaller the reconstruction error, the higher the performance of the algorithm, and the error approaches zero under ideal conditions. However, Yu Z. of Southwest Jiaotong University gave the lower bound of the reconstruction error of the input data, and gave a necessary condition for the reconstruction error of the input layer to reach an ideal state. When the input data is corrupted or noise is added, the reconstruction error of corrupted or noisy input data also has a lower bound. For the loss function of the autoencoder structure, such as formula (6), the pure data input by the autoencoder network can be expressed as $x$, the input pure data can be expressed as $x_{c}^{*}$, and the abstract parameters in the implicit part of the structure can be expressed as $h_{c}$. Formula (10) can be obtained through the encoder:

$$
f(x)=h_{c}
$$

Similarly, according to its symmetric structure, the reconstructed parameters can be encoded to obtain formula (11):

$$
h_{c}^{*}=f\left(x_{c}^{*}\right)
$$

The Taylor expansion of the Lagrangian remainder for the above formula can be obtained:

$$
f\left(x_{c}^{*}\right)=f(x)+\left(x_{c}^{*}-x\right)^{T} \nabla f\left[x+\rho\left(x_{c}^{*}-x\right)\right]
$$

where $\nabla f\left[x+\rho\left(x_{c}^{*}-x\right)\right]$ is the first derivative of the coding part, where $\rho \in(0,1)$ . The loss function is represented by $h_{c}$ and $h_{c}^{*}$ as:

$$
\begin{aligned}
& L\left(h_{c}, h_{c}^{*}\right)=L\left(h_{c}, f\left(g\left(h_{c}\right)\right)\right) \\
& =\left\|h_{c}^{*}-h_{c}\right\|=\left\|f\left(x_{c}^{*}\right)-f(x)\right\|^{2}
\end{aligned}
$$

Combining (12) can get:

$$
L\left(h_{c}, h_{c}^{*}\right)=\left\|\left(x_{c}^{*}-x\right)^{T} \nabla f\left[x+\rho\left(x_{c}^{*}-x\right)\right]\right\|^{2}
$$

Let $x_{c}^{*}-x=\left|\xi_{1}, \xi_{2}, \cdots, \xi_{m}\right|^{T}$, while denoting $\nabla f\left[x+\rho\left(x_{c}^{*}-x\right)\right]$ as a real matrix $A$. Then formula (14) can be expressed as:

$$
\begin{aligned}
& L\left(h_{c}, h_{c}{ }^{*}\right)=\left\|\xi_{1}, \xi_{2}, \cdots,\left.\xi_{m}\right|^{T} \cdot A\right\|^{2}=\sum_{j=1}^{n}\left(\sum_{i=1}^{m} a_{i j} \xi_{i}\right)^{2} \\
& \leq \sum_{j=1}^{n}\left[\left(\sum_{i=1}^{m} a_{i j}^{2}\right)\left(\sum_{i=1}^{m} \xi_{i}^{2}\right)\right]=\xi^{2} \cdot A^{2}
\end{aligned}
$$


Ideally, it is hoped that the reconstructed data and the produced data are as identical as possible, which can be expressed as:

$$
\lim _{x_{c}^{*} \rightarrow x} \nabla f\left[x+\rho\left(x_{c}^{*}-x\right)\right]_{F}^{2}=J_{f}(x)_{F}^{2}
$$

Formula (17) can be obtained from the above formula:

$$
L\left(x_{c}^{*}, x\right) \geq L\left(x_{c}^{*}, h_{c}\right) /\left\|J_{f}(x)\right\|_{F}^{2}
$$

Through the network structure of the convolutional autoencoder in Section 2.4, the $1^{*} 1000$-dimensional features of the signal are connected to the classifier through the encoded information of the fully connected layer. The confidence loss for classification represented by the Softmax [21, 22] function is shown in formula (18):

$$
L_{\text {cross }}=L_{S o f t}-\frac{1}{N} \sum_{N}^{i=1} \log \left(\frac{e^{h_{y_{i}}}}{\sum_{C} e^{h_{j}}}\right)
$$

The classification accuracy of the signal is evaluated by the normal cross-entropy loss. When this part of the network can complete the classification of the signal, it can be considered that the network has extracted significant information in each category, so the deconvolution network can be used to reconstruct the signal on this basis. In the previous coding structure, the position of the reserved value in each operation in the pooling layer is recorded, and the deconvolutional signal is expanded to the same dimension as the original according to the above position during deconvolution. The data format of the final structure output is $2 * 1024$. According to the above derivation, the sum of the reconstruction error and the classification error is guaranteed to be the smallest, so the error formula is obtained as follows:

$$
L_{\text {mypaper }}=\alpha L_{\text {classify }}+\|x-y\|^{2}-L\left(x_{c}^{*}, h_{c}\right) /\left\|J_{f}(x)\right\|_{F}^{2}
$$

where $L_{\text {classify }}$ is the Softmax cross-entropy loss above, and $\alpha$ is a hyperparameter. To balance the magnitude relationship between the two and accelerate the training convergence, this study sets this parameter to 0.1 .

\subsection{Network Structure}

According to the characteristics of the interference signal, it is considered that the interference signal needs to overlap with the communication signal in the timefrequency domain, and should have similar characteristics in the communication signal. Therefore, a convolutional encoder and a classification loss structure are proposed in this to generate interference signals, and the structure box is shown in Figure. 5.

Through the convolutional neural network in the above table, the signal is converted into features of dimension $1 * 1000$. For a signal with a length of $2^{*} 1024$, only a fully connected layer with a smaller dimension is required to describe the features. 
Table 1: Encoding part of the network structure

\begin{tabular}{|c|c|c|}
\hline Network Structure & Convolution Kernel Structure & Output Layer \\
\hline Input & T & $(2,1024)$ \\
\hline Convolutional layer (normalization) & $(2,2,128)$ & l \\
\hline ReLU/PReLU & 128 & / \\
\hline Convolutional layer (normalization) & $(1,2,128)$ & / \\
\hline ReLU/PReLU & 128 & / \\
\hline Pooling layer (max pooling) & $(1,2,128)$ stride $=2$ & / \\
\hline Convolutional layer (normalization) & $(1,2,256)$ & / \\
\hline ReLU/PReLU & 256 & 1 \\
\hline Convolutional layer (normalization) & $(1,2,256)$ & / \\
\hline ReLU/PReLU & 256 & / \\
\hline Pooling layer (max pooling) & $(1,2,256)$ stride $=2$ & / \\
\hline Convolutional layer (normalization) & $(1,2,512)$ & / \\
\hline $\operatorname{ReLU} / \mathrm{PReLU}$ & 512 & / \\
\hline Convolutional layer (normalization) & $(1,2,512)$ & / \\
\hline ReLU/PReLU & 512 & t \\
\hline Pooling layer (max pooling) & $(1,2,512)$ stride $=2$ & / \\
\hline Fully connected layer $($ dropout $=0.5)$ & $256 * 128 * 1$ & $1000 * 1$ \\
\hline Fully connected layer (dropout $=0.5)$ & $1000 * 1$ & $1000 * 1$ \\
\hline Classifier & $1000 * 3$ & $3 * 1$ \\
\hline
\end{tabular}

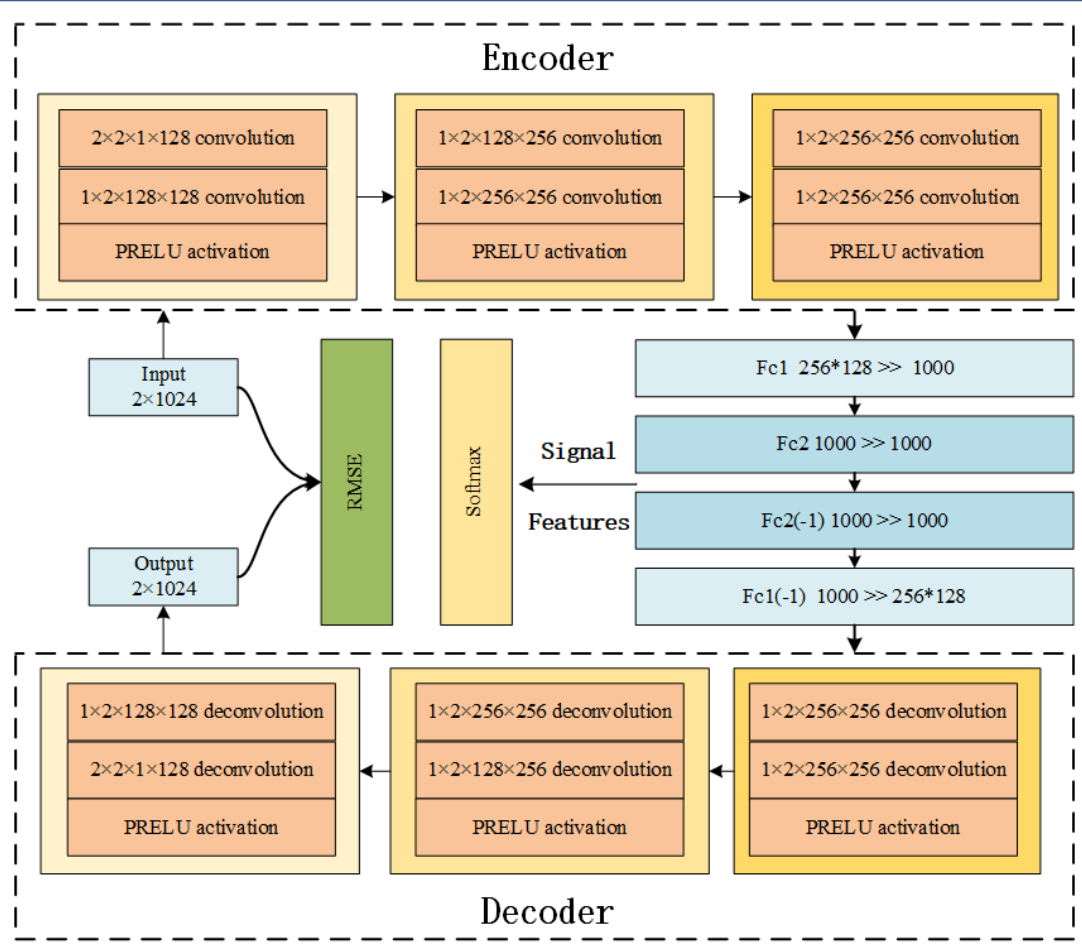

Figure 5: Algorithm block diagram

The purpose of using a longer dimension in this study is to provide a larger policy dimension space for subsequent generation of interference waveforms. The classifier is connected through the encoded information of the fully connected layer, and the upper part of the encoder is connected to the Softmax classifier, so that the neural network can extract the salient features of the communication signal in this way. At the same time, the fully connected layer is connected to the deconvolution network for decoding the communication signal. According to the symmetry of the struc- 
ture of the autoencoder and Table 1, the structure of the decoder can be reversely restored.

\section{Results and discussion}

To test the feasibility of the interference waveform generation technology proposed in this study, we divide the experiment into pattern analysis of generated signal and interference effect comparison experiment.

\subsection{Analysis of Algorithm Reconstruction Results}

To verify the subsequent interference effect, the reconstruction performance of the convolutional autoencoder needs to be tested first. In this experiment, the features with significant scores are kept unchanged while other non-salient features are changed. The $1 * 1000$-dimensional features are distributed in a ratio of 10:990, and the non-salient features or salient features are replaced with Gaussian random noise with the same energy to reconstruct the signal. The experimental results are illustrated in Figures 6 and 7.

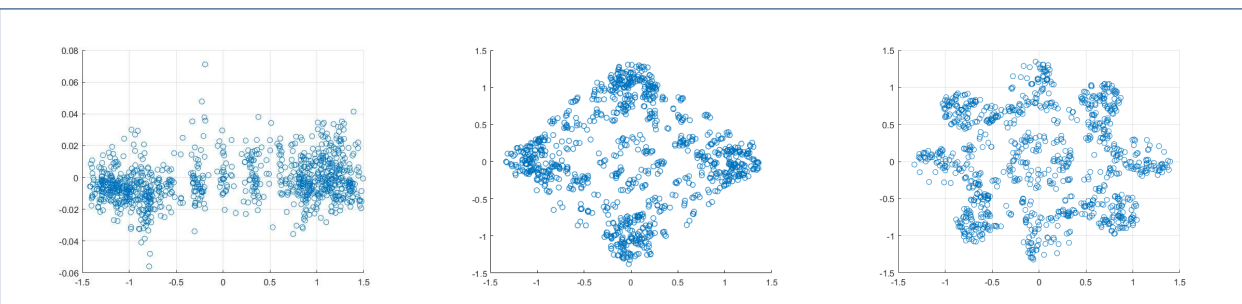

Figure 6: Constellation diagram of the generated interference waveform
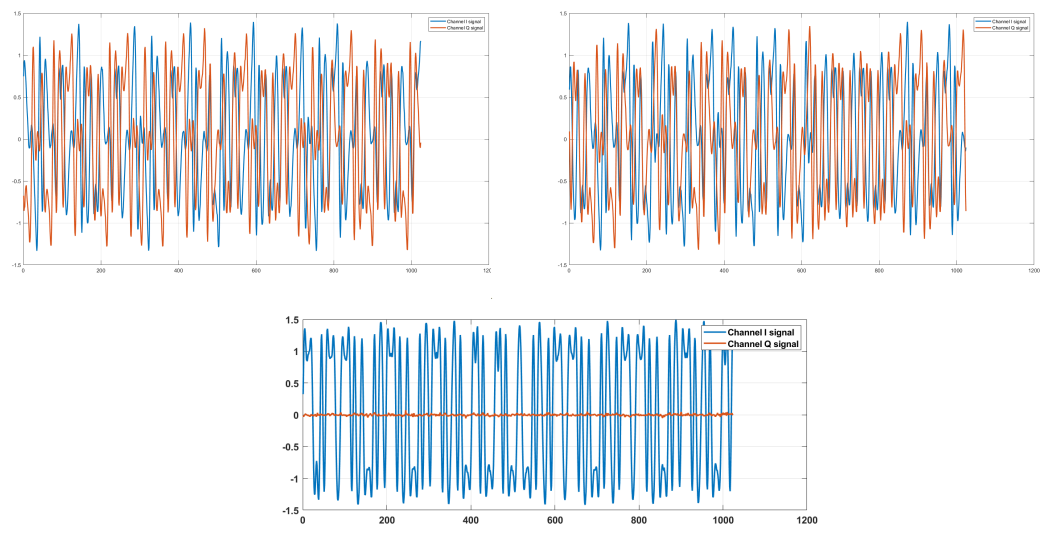

Figure 7: Randomly generated interference waveform

To detect the similarity between the interference signal and the communication signal more intuitively, the above figures illustrate the time domain I/Q waveform and constellation diagram of the generated interference.

The BSPK, QPSK, and 8PSK signals can be clearly distinguished from the constellation diagram, and the time-domain curve of the communication signal is 
smooth, which proves that the reconstructed interference waveform has a strong similarity with the communication waveform. However, there is a large gap between the constellation diagram of the BPSK signal and the original signal constellation diagram. This is because the unified mean square error is used in the training process, and the amplitude difference between the Q-channel signal of the BPSK signal and the other two types is too large.

\subsection{Analysis of the interference results of the algorithm}

This experiment adds perturbation and occlusion to the features in the fully connected layer, and verifies the interference performance of the waveform generated by the deconvolution. In order to compare and test the interference effect, the SNR is selected as $15 \mathrm{~dB}$, the communication mode is BPSK, QPSK, 8PSK, the SIR is $-1510 \mathrm{~dB}$, the step is $2 \mathrm{~dB}$, and a random string with a length of 1200 is used. The ASCII source code is used as a test sequence, and 100 tests are performed and the average value of the BER is taken. Select BPSK, QPSK and 8PSK three kinds of communication signal itself, noise amplitude modulation, Gaussian noise and other interference for comparison, and the interference effect curve is shown in Figure. 8.
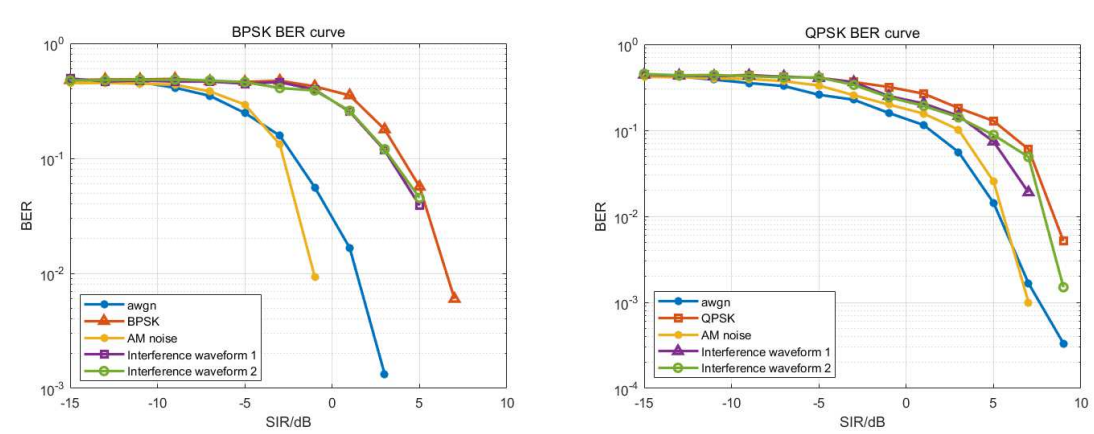

(a) BER Curve of BPSK Communica- (b) BER Curve of QPSK Communication System tion System

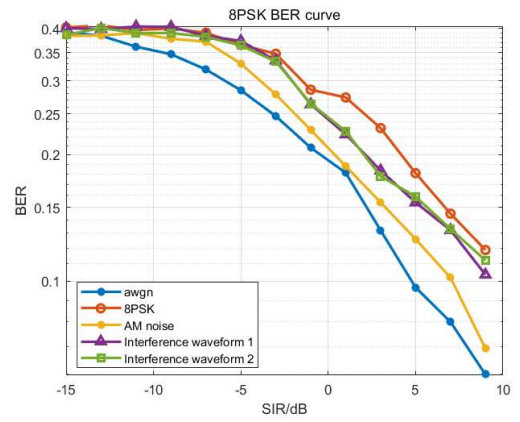

(c) BER Curve of 8PSK Communication System

Figure 8: BER curve under the generated interference waveform $(\mathrm{SNR}=15 \mathrm{~dB})$

In this experiment, the maximum weight of $1 \%$ of the fully connected layer is selected to apply Gaussian perturbation according to the two standards of mean and standard deviation, and the mean value of the fully connected layer is kept unchanged. The output interference signals are deconvolution interference waveform 1 
and deconvolution interference waveform 2 respectively. The low interference performance in BPSK communication is because a certain amount of energy is allocated to the Q-channel signal in the waveform reconstruction, resulting in the degradation of the interference performance. However, the BER of QPSK and 8PSK communication waveforms are both higher than noise amplitude modulation and Gaussian noise interference.

In summary, the method proposed in this study can generate an interference waveform that approximates the communication signal, and is better than the interference performance of Gaussian noise and noise amplitude modulation under different SIRs.

\section{Conclusion}

Aiming at the problem of signal management and control in the IoV, this study introduces the intelligent interference of communication signals into the signal processing of the IoV, and proposes a method for generating interference waveforms based on convolutional autoencoders. To ensure the applicability and validity of the experimental results, a complete vehicle network communication system is simulated and built in this study, and three communication signals of BPSK, QPSK and 8PSK are used for communication. In this study, a method based on convolutional autoencoder is proposed to extract high-order features of communication signals, and the required intelligent interference waveform is obtained by occluding and replacing the features on the fully connected layer. It can be seen from the simulated constellation diagram that the reconstructed interference waveform has a strong similarity with the communication waveform, which proves the similarity between the generated interference waveform and the original signal waveform. In addition, the bit error rate curve obtained by simulation can prove that the interference performance of the interference waveform generated in this study is better than that of Gaussian noise and noise amplitude modulation under different SIRs.

The modulation methods applied to the IoV signals are complex and diverse, and this study only focuses on three phase shift keying signals for communication system construction and intelligent interference. Therefore, in future research, the types of modulation methods included should be expanded, so as to achieve comprehensive control of IoV signals.

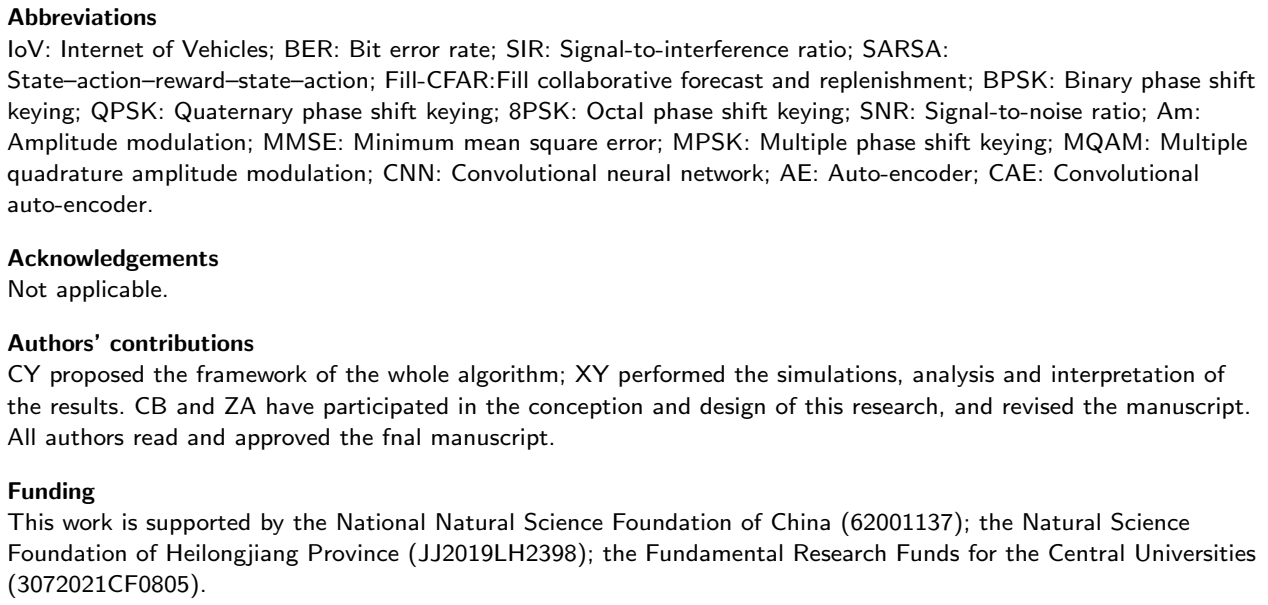


Availability of data and materials

Please contact author for data requests.

Ethics approval and consent to participate

Not applicable.

\section{Consent for publication}

The picture materials quoted in this article have no copyright requirements, and the source has been indicated.

Competing interests

The authors declare that they have no competing interests.

\section{Author details}

${ }^{1}$ College of Information and Communication Engineering, Harbin Engineering University, Harbin, China. ${ }^{2}$ Key Laboratory of Advanced Marine Communication and Information Technology, Ministry of Industry and Information Technology, Harbin Engineering University, Harbin, China.

\section{References}

1. Fu, E., Li, X., Yao, Z., Ren, Y., Wu, Y., Fan, Q.: Personnel emotion recognition model for internet of vehicles security monitoring in community public space. EURASIP Journal on Advances in Signal Processing 2021(1), $1-19$ (2021)

2. Yazdi, H.S., Lotfizad, M., Kabir, E., Fathy, M.: Clipped input rls applied to vehicle tracking. EURASIP Journal on Advances in Signal Processing 2005(8), 1-8 (2005)

3. Liu, X., Zhang, X.: Noma-based resource allocation for cluster-based cognitive industrial internet of things. IEEE transactions on industrial informatics 16(8), 5379-5388 (2019)

4. Liu, X., Zhai, X.B., Lu, W., Wu, C.: Qos-guarantee resource allocation for multibeam satellite industrial internet of things with noma. IEEE Transactions on Industrial Informatics 17(3), 2052-2061 (2019)

5. Wei, B., Zhang, L., Wang, K., Kong, Q., Wang, Z.: Dynamic scene deblurring and image de-raining based on generative adversarial networks and transfer learning for internet of vehicle. EURASIP Journal on Advances in Signal Processing 2021(1), 1-19 (2021)

6. Arróspide, J., Salgado, L., Nieto, M.: Video analysis-based vehicle detection and tracking using an mcmc sampling framework. EURASIP Journal on Advances in Signal Processing 2012(1), 1-20 (2012)

7. Xu, X., Wang, Y., Liu, J., Zhang, X.: Analysis on the achievement milestones and limitations of game theory. In: 2008 Chinese Control and Decision Conference, pp. 1214-1219 (2008). IEEE

8. Shafiee, S., Ulukus, S.: Capacity of multiple access channels with correlated jamming. In: Military Communications Conference, 2005. MILCOM 2005. IEEE (2005)

9. Kashyap, A., Basar, T., Srikant, R.: Correlated jamming on mimo gaussian fading channels. IEEE Transactions on Information Theory (2006)

10. Chang, L.: An information-theoretic study of ratio-threshold antijam techniques. thesis illinois univ (1985)

11. Amuru, S., Buehrer, R.: Optimal jamming against digital modulation. IEEE Transactions on Information Forensics \& Security 10(10), 2212-2224 (2015)

12. Farzamnia, A., Hlaing, N.W., Haldar, M.K., Rahebi, J.: Channel estimation for sparse channel ofdm systems using least square and minimum mean square error techniques. In: 2017 International Conference on Engineering and Technology (ICET) (2018)

13. Yang, C., Yang, F., Wang, Z.: Iterative minimum mean square error equalization for optical fiber communication systems. IEEE Photonics Technology Letters 19(19), 1571-1573 (2007)

14. Huang, L., Neely, M.J.: The optimality of two prices: Maximizing revenue in a stochastic communication system. IEEE/ACM transactions on networking 18(2), 406-419 (2009)

15. Uçkun, F., Özer, H., Nurbaș, E., Onat, E.: Direction finding using convolutional neural networks and convolutional recurrent neural networks. In: 2020 28th Signal Processing and Communications Applications Conference (SIU), pp. 1-4 (2020)

16. Zhou, Y., Amimeur, A., Jiang, C., Dou, D., Jin, R., Wang, P.: Density-aware local siamese autoencoder network embedding with autoencoder graph clustering. In: 2018 IEEE International Conference on Big Data (Big Data), pp. 1162-1167 (2018). IEEE

17. Xu, Q., Wu, Z., Yang, Y., Zhang, L.: The difference learning of hidden layer between autoencoder and variational autoencoder. In: 2017 29th Chinese Control And Decision Conference (CCDC), pp. 4801-4804 (2017). IEEE

18. Zhao, X., Han, X., Su, W., Yan, Z.: Time series prediction method based on convolutional autoencoder and Istm. In: 2019 Chinese Automation Congress (CAC), pp. 5790-5793 (2019). IEEE

19. Chun, C., Jeon, K.M., Kim, T., Choi, W.: Drone noise reduction using deep convolutional autoencoder for uav acoustic sensor networks. In: 2019 IEEE 16th International Conference on Mobile Ad Hoc and Sensor Systems Workshops (MASSW) (2019)

20. Lee, H., Kim, J., Kim, B., Kim, S.: Convolutional autoencoder based feature extraction in radar data analysis. In: 2018 Joint 10th International Conference on Soft Computing and Intelligent Systems (SCIS) and 19th International Symposium on Advanced Intelligent Systems (ISIS), pp. 81-84 (2018). IEEE

21. Rao, Q., Yu, B., He, K., Feng, B.: Regularization and iterative initialization of softmax for fast training of convolutional neural networks. In: 2019 International Joint Conference on Neural Networks (IJCNN), pp. 1-8 (2019). IEEE

22. Hussain, M.A., Tsai, T.-H.: An efficient and fast softmax hardware architecture (efsha) for deep neural networks. In: 2021 IEEE 3rd International Conference on Artificial Intelligence Circuits and Systems (AICAS), pp. 1-4 (2021). IEEE 